\title{
XII CONGRESO INTERNACIONAL DE LA VIVIENDA/CHILE
}

\author{
(XII INTERNATIONAL CONGRESS ON HOUSING/CHILE)
}

M. ${ }^{a}$ José Escorihuela, Lcda. en Ciencias Físicas IETCC/CSIC

Madrid

\section{RESUMEN}

En este articulo se resume el desarrollo del XII Congreso Internacional de la Vivienda, celebrado en Viña del Mar (Chile) en marzo de 1985, y que ha tenido como tema central: "Nuevos Métodos de Construcción y Financiamiento".

Los trabajos presentados han tratado de dar soluciones técnicas, sociales y financieras al problema de la vivienda, especialmente en los paises en desarrollo.

La participación española ha consistido en dos trabajos, uno sobre la problemática concreta de las condensaciones en interiores de muros, y otro que expone un método gráfico que permite simplificar los procesos para adquirir mavor conocimiento de la radiación solar recibida sobre los distintos elementos de un edificio, con el fin de conseguir un mejor aprovechamiento de este tipo de energía y así obtener un mayor confort en el habitat humano.
SUMMARY

In this work the development of the XIIth International Congress on Housing, held in Viña del Mar (Chile) on March 1985, is summarized. Its central topic was: "New Construction and Financing Methods".

The presented papers intended to bring technical, social and financial solutions to housing problems, specially in developing countries.

Spanish participation include two works, one about the particular problem of the inner condensation in walls and another where it is showed a graphic method which allows to simplify of solar radiation in the diverse elements of a building, with the purpose to obtain a better use of this kind of energy, so as to reach a higher confort in human habitat.

\section{GENERALIDADES}

El XII Congreso Internacional de la Vivienda ha tenido lugar en Viña del Mar (Chile), y ha sido convocado y organizado bajo los auspicios de la International Association for Housing Science (IAHS); de la Florida International University (FIU) de Miami (USA), y del Departamento de Obras Civiles de la Universidad Técnica Federico Santa María (UTFSM) de Valparaíso (Chile).

El Comité organizador estaba presidido por el doctor O. Ural, director del IAHS, y por el profesor ingeniero R. Galindo, director del Departamento de Ingeniería Civil de la UTFSM. Como Secretario del Congreso actuó el profesor ingeniero C. Aguirre, del Departamento de Obras Civiles de la UTFSM.

Las diferentes sesiones se han desarrollado en el hotel O'Higgins de Viña del Mar, durante los días 25 al 28 de marzo de 1985, ambos inclusive, con un horario de mañana y tarde.
Cada sesión estaba presidida por un presidente, un co-presidente y los expositores que en ella iban a presentar sus trabajos.

Los idiomas oficiales han sido el español y el inglés, con servicio de traducción simultánea.

\section{OBJETIVOS}

El fin principal ha sido el dar ocasión a los interesados en el área de la edificación (técnicos, administradores, financieros, empresarios) de exponer y conocer nuevos métodos de construcción y financiamiento (tema central del Congreso), así como proporcionar la oportunidad de establecer y afianzar contactos, de intercambiar información, etc., sobre el momento actual y el futuro del campo de la vivienda. 


\section{SESION INAUGURAL}

En esta sesión, que contó con la presencia de diversas autoridades políticas y.científicas, se resaltó el problema del crecimiento de la población mundial, de los índices de costes y de la necesidad de una política de la vivienda y de la economía.

Se hizo mención de los efectos del terremoto que recientemente (3 de marzo de 1985) padeció Chile, de los daños causados en la edificación y de la ocasión que se ofrecía de observarlos «in situ» para así poder contribuir con un estudio posterior a la solución de los problemas planteados.

Se expresó la esperanza de que las ideas, teorías y datos que se iban a exponer a lo largo de estos días se confrontaran con situaciones concretas como son las fuerzas de la naturaleza y las justas aspiraciones de los países que luchan por su desarrollo.

\section{TRABAJOS Y ASISTENCIA}

Como se observa en el Cuadro 1, se enviaron al Congreso casi 80 trabajos de unos 30 países, habiéndose recibido al comienzo del mismo los textos completos de la mayoría de ellos en una publicación de dos volúmenes.

El total de personas inscritas ha sido cerca de 100 , procedentes de 14 países.

Durante el transcurso de las sesiones se han expuesto 26 comunicaciones correspondientes a 9 de los países asistentes.

Estas comunicaciones expuestas han tratado principalmente sobre:

- Nuevas alternativas de solución al problema de la vivienda mediante el estudio de nuevos métodos o la mejora de los existentes de manera que se aprovechen al máximo los recursos disponibles en cada uno de los países del mundo.

- La necesidad de una colaboración mundial efectiva para reducir el déficit de viviendas en los países en desarrollo y la conveniencia de buscar nuevas tecnologías para la vivienda social.

En este sentido, el arquitecto chileno, doctor Larrain, explicó que la Unión Internacional de Arquitectos (UIA) se ha propuesto el empeño de la convocatoria de un Concurso Mundial con el fin de elaborar un Plan Internacional de Construcción Social, y ha encomendado a su Sección Española, con sede en Madrid, presidida por el arquitecto español don Rafael de la Hoz, la dirección y asesoría del mismo. El concurso no se ha lanzado porque falta su financiación.

\begin{tabular}{|c|c|c|c|}
\hline \multicolumn{4}{|c|}{ Cuadro 1} \\
\hline Países & $\begin{array}{c}\text { Trabajos } \\
\text { presentados }\end{array}$ & Expuestos & $\begin{array}{l}\mathrm{N} .{ }^{\circ} \text { personas } \\
\text { inscritas }\end{array}$ \\
\hline Alemania & - & - & 1 \\
\hline Arabia Saudita & 4 & - & - \\
\hline Árgentina & 2 & - & 8 \\
\hline Austria & 1 & 1 & 1 \\
\hline Bolivia & - & - & 11 \\
\hline Brasil & 3 & - & - \\
\hline Canadá & 1 & - & - \\
\hline Chile & 12 & 10 & 52 \\
\hline Costa Rica & - & - & 2 \\
\hline R. Dominicana & 1 & - & - \\
\hline Ecuador & 1 & 1 & 2 \\
\hline Egipto & 8 & - & - \\
\hline España & 2 & 2 & 2 \\
\hline Estados Unidos & 10 & 5 & 9 \\
\hline Ghana & 1 & - & - \\
\hline Guatemala & 1 & - & 1 \\
\hline India & 5 & 1 & 1 \\
\hline Irán & 1 & - & - \\
\hline Japón & 1 & - & - \\
\hline Jordania & 1 & - & - \\
\hline Nigeria & 6 & 3 & 3 \\
\hline Reino Unido & 1 & - & - \\
\hline Rumania & 2 & 一 & 一 \\
\hline Singapur & 5 & 2 & 2 \\
\hline Sudáfrica & 1 & 1 & 2 \\
\hline Trinidad & 2 & - & - \\
\hline Turquía & 4 & - & - \\
\hline 27 & 76 & 26 & 97 \\
\hline
\end{tabular}

Por ello, desde estas páginas, hago un llamamiento para que las personas, entidades, organismos, etc., públicos y privados que puedan contribuir de alguna manera a la convocatoria y financiación de dicho Concurso, presten su apoyo y se animen a colaborar con el fin de dar un paso que puede ser muy importante para afirmar la paz mundial.

- La conveniencia de estudiar y planificar sistemas de vivienda que contribuyan a la interacción entre grupos y clases, de manera que todas las personas puedan desarrollar sus emociones y capacidades en igualdad de ambiente.

\section{PARTICIPACION ESPAÑOLA}

La única participación activa española ha sido la del Instituto Eduardo Torroja de la Construcción y del Cemento de Madrid, y ha consistido en dos trabajos:

Uno de ellos: "Estudio de soluciones constructivas tradicionales de ladrillos cerámicos en el problema de condensación en interiores», del que es autor M. Olaya, jefe del equipo de «Ahorro de Energía en la Edificación» del IETCC, del Consejo Superior de Investigaciones Científicas. Madrid. 





En este trabajo se analiza la respuesta teórica de distintas combinaciones de muros construidos con los diferentes formatos de ladrillos fabricados en España a los problemas de condensaciones en el interior de los mismos, considerando la solución llamada directa (consistente tradicionalmente en un elemento grueso exterior de ladrillo cerámico perforado, una cámara de aire y un elemento delgado interior de ladrillo hueco), y la llamada invertida (de la anterior).

La legislación española contempla, entre otras prescripciones que deben cumplir los edificios, los aspectos relativos a condensaciones en cerramientos exteriores.

Se calculan, en primer lugar, los gradientes térmicos de temperatura seca y en base a estas temperaturas, mediante un ábaco psicrométrico, se estiman los valores de presión de saturación en las distintas zonas del muro.

A continuación se calculan las presiones de vapor correspondientes a unas condiciones higrotérmicas dadas $\left(18{ }^{\circ} \mathrm{C}\right.$ para interior $\mathrm{y}-2,0,3,5,10^{\circ} \mathrm{C}$ para exterior), y para los gradientes térmicos obtenidos anteriormente en las distintas zonas del muro.

Si estos valores de presión de vapor son superiores a los de presión de saturación, se supone que en esa zona existe condensación. Se realizan cálculos para las soluciones llamadas directa e invertida.

Se contempla un caso particular de solución constructiva y se observa, al comparar los valores, que en la solución directa se produce condensación en zonas del interior de dicha solución, ya que la presión de vapor en ellas es superior a la de saturación, lo cual no sucede en el caso de la solución inversa.

Por tanto, en la problemática concreta de las condensaciones, en interiores de muros, se puede rehabilitar la idea de invertir los elementos de muros tradicionales.

El otro trabajo, «Representaciones gnomónicas del camino aparente del sol. Aplicaciones», del que son autores Bernardo Torroja y M. J. Escorihuela, físicos del Instituto Eduardo Torroja del Consejo Superior de Investigaciones Científicas de Madrid.

En él se desarrolla un método gráfico que permite determinar el soleamiento en el interior y exterior de edificaciones, así como las sombras por ellas producidas.

Se basa en la "Proyección Gnomónica» del camino aparente del sol y resulta de proyectar este camino sobre un plano tangente, en el zenit del observador, a la bóveda celeste. El punto de proyección es el centro de dicha bóveda.
De esta manera se obtienen unos gráficos en los que aparecen las trayectorias solares particularizadas para un día concreto del año y para diferentes valores $(r=1,2 \ldots 8)$ del radio $r$ de la bóveda celeste (ver gráfica en pág. anterior).

En el origen del gráfico confluyen una familia de rectas que representan las líneas horarias.

En la exposición se ha explicado particularmente el caso del soleamiento en el interior de una habitación producido por el hueco de una ventana:

- Se dibuja a determinada escala la planta de la habitación con las paredes abatidas sobre el plano horizontal y, en línea con la pared correspondiente, se señala la amplitud de la ventana indicando su orientación.

- Se toma el gráfico correspondiente a la altitud de situación del edificio y el día elegido y a la misma escala que la del plano de la habitación.

- Sobre el vértice del gráfico se sitúa el punto extremo (en el plano) de la amplitud de la ventana, orientándolo previamente, de manera que el norte del plano «mire» al sur del gráfico.

- Para una hora determinada, se buscan sucesivamente los puntos intersecciones de la línea horaria con las curvas que representan alturas correspondientes a las distancias al suelo de las partes superior e inferior de la ventana; estos puntos serían los extremos de los rayos solares si el suelo fuese indefinido.

- Si uno de estos puntos cae fuera del recinto limitado por las cuatro paredes, se considera el punto intersección de la línea horaria con la pared sobre la que incide y se observa a qué altura corresponde la curva que pasa por tal punto. A esta altura el rayo incidirá (con relación a la parte superior de la ventana) sobre la pared.

- Recorriendo, a continuación, toda la dirección de la amplitud de la ventana sobre el gráfico gnomónico, obtendríamos, de forma sencilla, las distancias a que incidirían los rayos solares en direcciones perpendiculares a la pared.

- Se procedería de igual manera para todas las horas del día.

- De esta manera conoceríamos las características del soleamiento producido por el hueco de la ventana sobre el suelo y sobre las paredes.

- Utilizando los gráficos de los días extremos del año podríamos, por tanto, llegar a determinar la variación del soleamiento a lo largo del año.

Los "Artificios Gráficos», entre los que se encuentran los Gráficos Gnomónicos, simplifican mucho los procesos para definir las características solares, la forma, dimensiones, etc., del sombreado o soleamiento, y contribuyen así a proyectar la mejor orientación, forma y diseño de los edificios en función de que cumplan las exigencias de captación, 
acumulación y distribución de energía solar para obtener un mejor confort en la vivienda.

Las dos exposiciones españolas fueron realizadas por M. J. Escorihuela debido a ser el único miembro designado por el Instituto Eduardo Torroja para trasladarse a Viña del Mar (Chile) con el fin de participar en este Congreso.

\section{SESION DE CLAUSURA}

En esta sesión, plenaria y última del Congreso, se organizó un Foro Panel coordinado por: O. Ural presidente del IAHS, y R. Galindo, presidente del Comité Organizador; y del que formaban parte: $C$. Aguirre, profesor de la UTFSM; R. Krafenbauer, miembro directivo del IAHS; G. Nedeljkov, del Colegio de Arquitectura de la Universidad Técnica de Berlín; R. Mills-Tettey, de la Universidad de Ife, Nigeria; y M. ${ }^{a}$ J. Escorihuela, del Instituto Eduardo Torroja, Madrid (España); en el que se trató sobre aspectos técnicos de las edificaciones dañadas por el sismo, y se hizo un análisis sobre el déficit mundial de viviendas y sobre el concepto de vivienda social en sus acepciones técnicas y socio-económicas. Lo importante de este tipo de viviendas es que deben satisfacer las necesidades y aspiraciones del grupo familiar.

Esta sesión también dio su respaldo al proyecto de un concurso mundial sobre «Nuevas tecnologías para la vivienda social».

El doctor Nedeijkov invitó a los presentes a participar en el próximo "XIII IAHS World Congress on Housing», que tendrá lugar del 6 al 10 de abril de 1987 en Berlín (Alemania), con el tema central: «Innovations in Sciences \& Technologies for the Future».

En el acto de clausura, la Universidad Federico Santa María de Valparaiso (Chile) hizo entrega, por medio de los doctores Galindo, Ural y Krapfenbauer, de unos diplomas de participación a los asistentes y expositores, al término del cual se dio por finalizado el XII Congreso Internacional de la Vivienda.

\section{VISITAS}

Entre los actos científicos del Congreso se organizaron visitas a las zonas de Viña del Mar y alrededores afectadas por el sismo que sufrió el país, previa sesión especial en la que se mostraron diapositivas sobre diversas edificaciones de distintas localidades chilenas en las que se apreciaban las consecuencias más o menos graves de dicho sismo.

También se visitaron las instalaciones académicas y diversos laboratorios de la UTFSM de Valparaiso, cuya estructura se basa en tres Facultades: la de Ingeniería, la de Ciencia y la de Economía y Administración. Se puso especial atención en los Departamentos de Ingeniería y Construcción Civil de la Facultad de Ingeniería, cuya misión furidamental es la docencia e investigación en el área de la construcción y sus materiales.

Al final del Congreso la autora de este artículo visitó, invitada por el doctor Alliende, director de la Escuela de Construcción Civil de la Universidad Católica de Chile en Santiago, el «Campus San Joaquín» donde está situada esta Escuela, y junto a él y otros profesores de la Universidad recorrió algunas naves de ensayo y laboratorios que desarrollan investigación en Ciencias de la Ingeniería y en aplicaciones tecnológicas. Se visitó también la moderna biblioteca centralizada para toda la Universidad, que con su amplio material gráfico, fotográfico y de video, facilita la formación académica de los alumnos.

Asimismo, se realizó en la ciudad de Santiago una visita técnica al Instituto de Investigaciones y Ensayos de Materiales (IDIEM), Centro de la Universidad de Chile que realiza investigación, asesora y apoya a instituciones públicas y privadas, industrias de la construcción, empresas de ingeniería, etc., en diversos aspectos relacionados con el estudio y control de calidad de materiales de construcción, de estructuras y elementos estructurales y constructivos, control de obras, acústica de locales, aislamiento térmico, etc., acompañada del doctor Lamana, director del Centro, así como del doctor Rodríguez, quien en este momento está especialmente interesado sobre el tema de diseño solar pasivo.

\section{CONCLUSIONES}

Se hace notar la disminución de países asistentes en relación con los que previamente habían enviado trabajos a este Congreso, circunstancia que tal vez debe atribuirse en parte al terremoto que 20 días antes del comienzo de esta reunión internacional afectó al país anfitrión, Chile.

Se ha observado la gran preocupación mundial en el tema de escasez de viviendas con relación al incremento de la población y a la dificultad de adquirirlas debido al costo de las mismas, ya que los temas desarrollados trataron principalmente de dar soluciones técnicas, sociales y financieras a este problema.

Finalmente se quiere resaltar el espíritu de colaboración que ha existido entre los asistentes de los distintos países y la inquietud de contribuir con sus conocimientos y esfuerzos a mejorar los temas relacionados con el campo de la vivienda. 